\title{
EL DECENIO DE LA EDUCACIÓN PARA EL DESARROLLO SUSTENTABLE EN LA UNIVERSIDAD DE SONORA
}

\author{
The decade of education for sustainable \\ development at the university of sonora
}

\section{EPISTEMUS}

ISSN: 2007-8196 (electrónico)

ISSN: 2007-4530 (impresa)

Marco Alberto a la Torre Islas 1

Andrea Zavala Reyna 2

Juana Alvarado Ibarra ${ }^{3}$

Luis Eduardo Velázquez Contreras 4

Recibido: 15 de marzo de 2015,

Aceptado: 22 de febrero de 2016

Autor de Correspondencia:

Dra. Andrea Zavala Reyna

Correo: azavala@industrial.uson.mx

\section{Resumen}

El presente escrito muestra los resultados obtenidos con el cálculo de indicadores para conocer la contribución que ha tenido la Universidad de Sonora, Unidad Centro, a la Educación para la Sustentabilidad, de acuerdo con los principios del Decenio de las Naciones Unidas de la Educación para el Desarrollo Sostenible, establecido por la UNESCO del 2005-2014. Destaca el hecho de que $100 \%$ de los programas de la Universidad de Sonora asumen el compromiso de incorporar la perspectiva ambiental y de sustentabilidad al quehacer de la universidad como una política institucional; así como que el 33.33\% de las divisiones operan bajo un sistema de gestión ambiental, entre otros importantes indicadores.

Palabras clave: Decenio de la educación, Universidad de Sonora, indicadores para la educación, desarrollo sustentable.

\section{Abstract \\ This paper presents the results obtained with the calculation of indicators to understand the contribution that has had the University of Sonora, unit center, to the Education for Sustainability in accordance with the principles of The UN Decade of Education for Sustainable Development (DESDE 2005-2014), established by UNESCO, highlights the fact that 100\% of the programs of the University of Sonora incorporate environmental sustainability perspective to the work of the university as an institutional policy; and the $33.33 \%$ of the divisions operate under a system of environmental management, among other important indicators.}

Keywords: Decade of education, University of Sonora, indicators for education, sustainable development.

1 Posgrado en Sustentabilidad, Universidad de Sonora / Correo: beto180588@hotmail.com 


\section{INTRODUCCIÓN}

La Asamblea General de las Naciones Unidas crea el Ilamado Decenio de las Naciones Unidas de la Educación para el Desarrollo Sostenible 2005-2014 [1], como una herramienta para afrontar el deterioro ambiental que ha puesto la existencia de los seres vivos en crisis, al afectar asentamientos humanos, territorios deshabitados y espacio exterior [2]; incluye la visión de un mundo donde todos tengan la oportunidad de beneficiarse de la educación y aprender los valores, comportamientos y estilos de vida necesarios para un futuro sustentable y para la transformación positiva de la sociedad [3], basados principalmente en el concepto de Educación para el Desarrollo Sustentable (EDS), el cual puede ser definido como una combinación de los principios existentes y aún por identificar de conocimientos, habilidades, perspectivas y valores que se organizan en torno a los conceptos y temas de sustentabilidad [4].

En este sentido, el país cuenta con el Consejo Nacional de Educación Ambiental para la Sustentabilidad, que es un órgano que fomenta el Decenio de las Naciones Unidas de la Educación para el Desarrollo Sostenible 2005-2014, a través de la Estrategia de Educación Ambiental para la Sustentabilidad en México [5]; dicha estrategia argumenta que la educación y formación de recursos humanos para la EDS es uno de los principales asuntos estratégicos que inciden en todas las modalidades de educación y comunicación ambientales, por lo que establece entre otras acciones cruciales la reestructuración de la currícula de las instituciones de educación superior que requieran competencias profesionales en EDS [6].

Considerando lo anterior, la EDS debe ser incluida en un número mayor de áreas disciplinares y en todos los niveles de la enseñanza [7], donde las instituciones de educación superior deben ser el principal agente de cambio que proporcione respuestas a los problemas y a los retos de la sociedad actual [8], ya que su mayor compromiso es preparar a las nuevas generaciones en el conocimiento de saberes universales, vinculados a los conocimientos étnicos y culturales propios de las regiones del país [9].

Ante los problemas ambientales, sociales y económicos que tenemos en la actualidad surge la necesidad dentro de las Instituciones de Educación Superior (IES) de comprometerse con la difusión e implementación de acciones que promuevan la cultura de la sustentabilidad dentro de sus campus universitarios, lo cual permita formar egresados que contribuyan a crear estilos de vida más sustentables con la sociedad. La Universidad de Sonora, no está exenta de este compromiso, por lo que ha realizado acciones encaminadas a prevenir, reducir y/o eliminar los riesgos ambientales y ocupacionales generados por sus actividades sustantivas y administrativas. El presente documento muestra los resultados obtenidos de un diagnóstico que se hizo a través de indicadores que midieron la contribución al desarrollo sustentable en la Universidad de Sonora.

\section{METODOLOGÍA}

El presente trabajo posee un enfoque cuantitativo, ya que se generó información numérica con el uso de los indicadores para medir la contribución de la Universidad de Sonora en el tránsito hacía la sustentabilidad, según los principios del Decenio de las Naciones Unidas de la Educación para el Desarrollo Sostenible de la UNESCO.

Para la realización del diagnóstico fue necesario estimar indicadores que midieran la contribución al desarrollo sustentable en la Universidad de Sonora (UNISON); dichos indicadores se calcularon a través de la adecuación de varios de ellos para medir la contribución de las Instituciones de Educación Superior a la Sustentabilidad, establecidos por el Consorcio Mexicano de Programas Ambientales Universitarios para el Desarrollo Sustentable (COMPLEXUS) y apegados a lo establecido por el Decenio de las Naciones Unidas de la Educación para el Desarrollo Sostenible. La investigación se desarrolló en la unidad centro de la UNISON, la cual se encuentra localizada en la ciudad de Hermosillo, Sonora.

\section{RESULTADOS}

A continuación se presentan los resultados obtenidos por cada uno de los indicadores que muestran la contribución a la sustentabilidad de la Universidad de Sonora. En la figura 1 se pueden ver los resultados para el indicador 1: Incorporación de la perspectiva ambiental y de sustentabilidad al quehacer de la universidad como una política institucional.

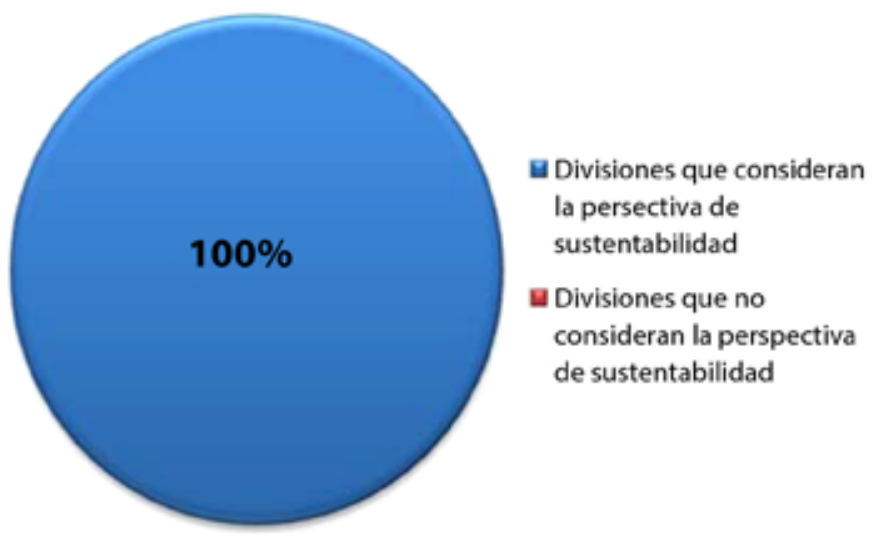

Figura 1. Incorporación de la perspectiva
ambiental y de sustentabilidad.

Según la política de sustentabilidad, la Universidad de Sonora asume plenamente los compromisos que rigen el Decenio de las Naciones Unidas de la Educación para el Desarrollo Sostenible [10].

Para el cálculo del indicador, se consideraron el número de Divisiones Académicas de la UNISON que consideran los principios de la sustentabilidad. Se tienen 6 Divisiones académicas y se encontró que el $100 \%$ de las divisiones consideran la perspectiva ambiental y de sustentabilidad como una política institucional. 
La figura 2 muestra los números obtenidos para el indicador 2: Sistemas de gestión ambiental implementados en la institución.

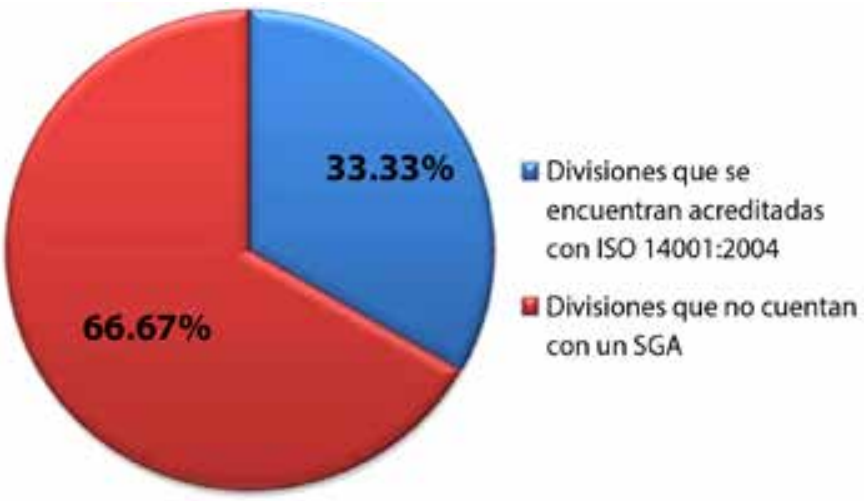

Figura 2. Sistemas de gestión ambiental implementados en la institución.

Recientemente la Universidad de Sonora, unidad centro, obtuvo la re-certificación y certificación de las divisiones de Ingeniería y de Ciencias Biológicas y de la Salud, respectivamente [11] bajo el esquema ambiental ISO 14001:2004. Para el cálculo del indicador, se dividió el número de divisiones académicas de la UNISON, Unidad Centro que se encuentran certificadas bajo el estándar internacional ISO 14001:2004 entre el total de divisiones, y posteriormente se multiplicó por 100 , obteniéndose que el $33.33 \%$ de ellas se encuentra acreditada por un Sistema de gestión ambiental.

La figura 3 muestra los resultados obtenidos en el indicador 3: Perspectiva de género en los diferentes niveles organizacionales de las IES y equidad de género en puestos de toma de decisiones.

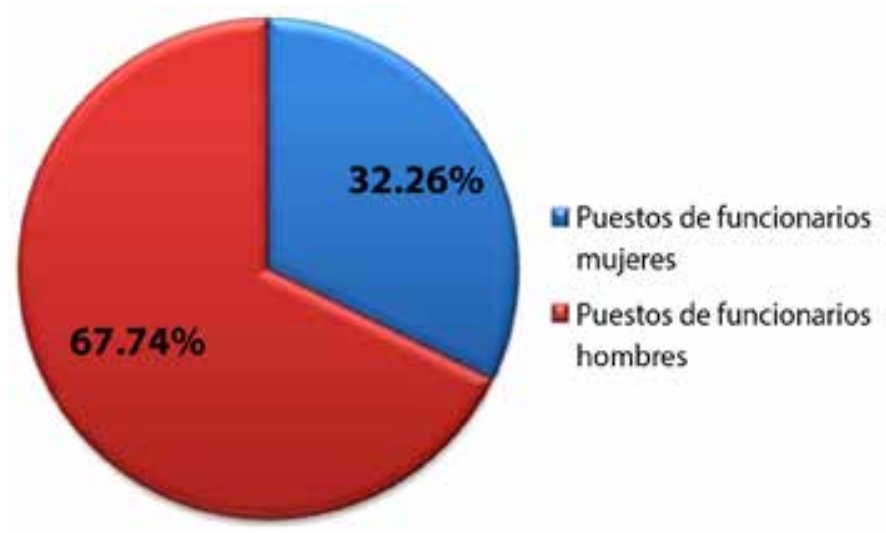

Figura 3. Perspectiva de género.

Con base en el directorio de funcionarios de la Universidad de Sonora, la Unidad Centro tiene en total 62 puestos de toma de decisiones importantes, de los cuales 20 son del sexo femenino [12]. Para el cálculo del indicador, se dividieron el número de puestos de toma de decisiones ocupados por mujeres entre el número total de espacios organizacionales de toma decisiones y se multiplicó por 100 , obteniéndose que el $32.26 \%$ de los puestos funcionarios en la Universidad de Sonora de la unidad centro son ocupados por mujeres.

En la figura 4 se observan los resultados correspondientes al indicador 4: Planes de estudio de nivel licenciatura que han incorporado la perspectiva ambiental y de sustentabilidad.

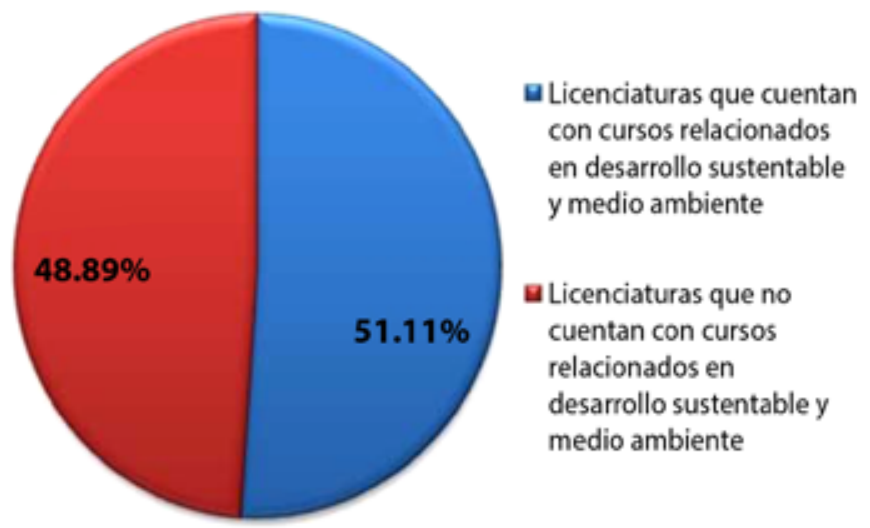

Figura 4. Planes de estudio con cursos relacionados al Desarrollo sustentable.

Actualmente la Universidad de Sonora en la unidad centro ofrece en total 45 licenciaturas, de las cuales 23 incluyen en sus planes de estudio alguna materia relacionada con el medio ambiente y el desarrollo sustentable [13]. Lo cual, según el indicador, el $51.11 \%$ de las licenciaturas ofertadas en la Unidad Centro de la UNISON cuenta con algún curso relacionado con dicho indicador.

Los resultados correspondientes al indicador 5: Planes de estudio de nivel licenciatura bajo el enfoque de competencias profesionales, se muestran en la figura 5 .

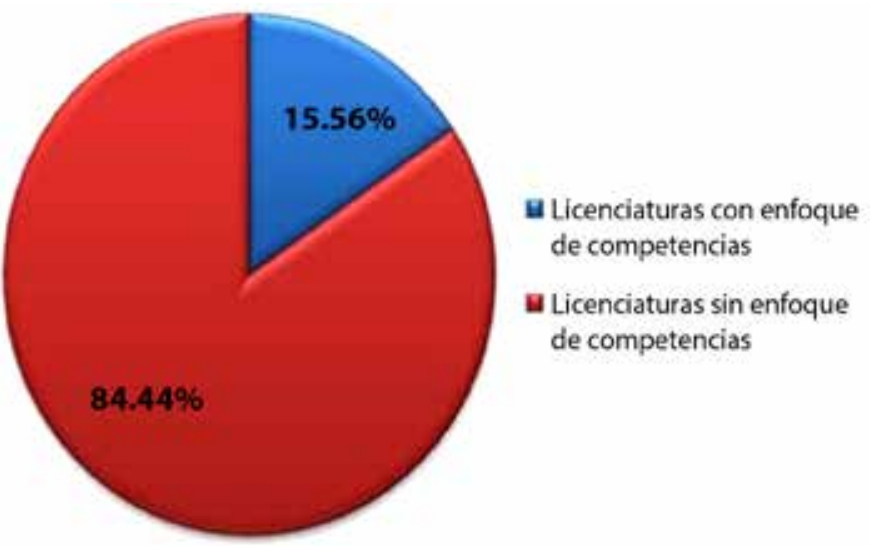

Figura 5. Licenciaturas bajo el enfoque de competencias.

Según la Dirección de Innovación Educativa de la Universidad de Sonora, de las 45 licenciaturas que se 


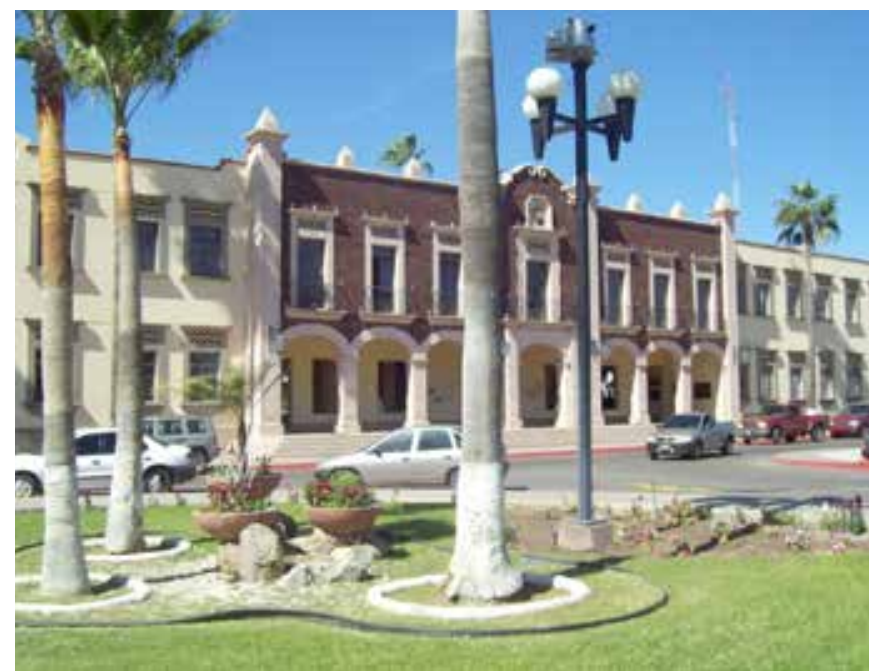

ofrecen en el campus centro, 9 están estructuradas bajo el enfoque de competencias [14]. Para el cálculo del indicador, se dividieron el número de planes de estudio de nivel licenciatura que sean reconocidos por la UNISON bajo el enfoque de competencias entre el total de planes de estudio de nivel licenciatura y se multiplicó por 100. El $15.56 \%$ de las licenciaturas ofertadas en la Unidad Centro de la UNISON se encuentran estructuradas bajo el enfoque de competencias.

La figura 6 muestra los resultados para el indicador 6: Proyectos de servicio social en medio ambiente y sustentabilidad.

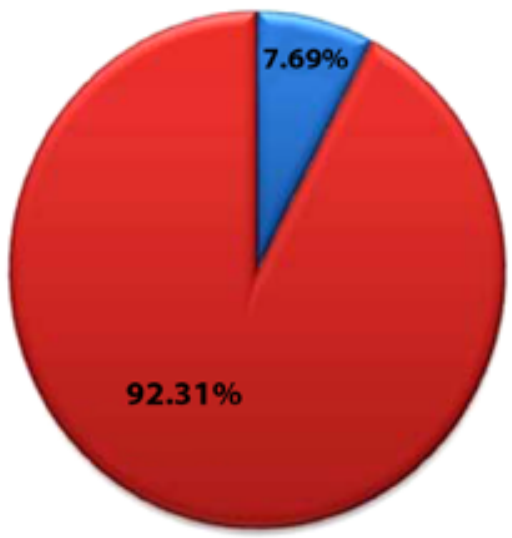

a Proyectos de servicio social relacionados con el medio ambiente y desarrollo sustentable

Figura 6. Proyectos de servicio social con enfoque de medio ambiente y sustentabilidad.

Según la Dirección de Servicios Estudiantiles de la Universidad de Sonora, el campus centro aprobó en el periodo enero - mayo del 2014 un total de 468 proyectos de servicio social, de los cuales 36 estaban relacionados con el desarrollo sustentable y medio ambiente [15]. Por lo que, realizando el cálculo de número de proyectos de servicio social relacionados en temas ambientales y de sustentabilidad entre el total de proyectos de servicio social y multiplicado por 100 , da que un $7.69 \%$ de los proyectos de servicio social universitarios aprobados por la Universidad de Sonora, Unidad Centro en el ciclo 2014-1 se encuentran relacionados con el indicador.

Por último, la figura 7 nos permite visualizar los resultados del indicador 7: Prestación de servicios profesionales en cuidado del medio ambiente y desarrollo sustentable.

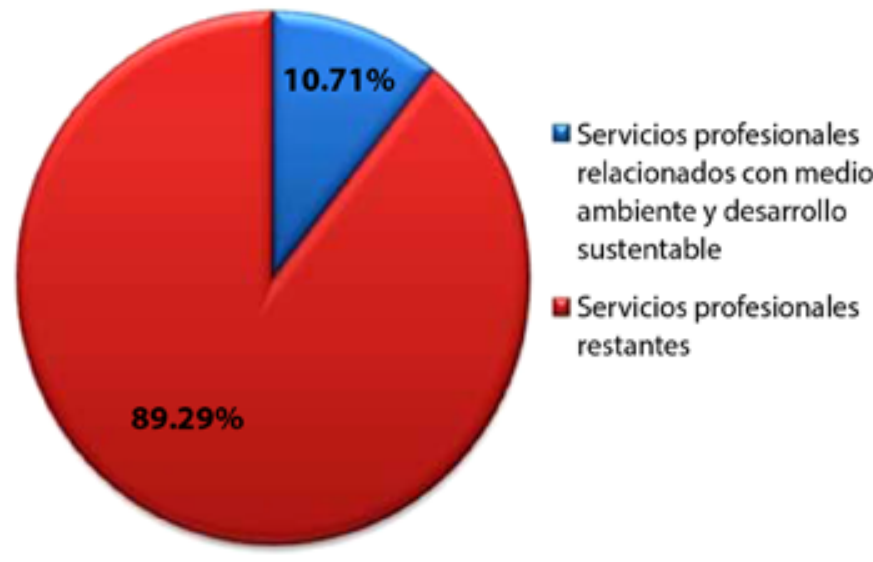

Figura 7. Prestación de servicios profesionales en cuidado del medio ambiente y desarrollo sustentable.

Según la Dirección de Vinculación y Difusión de la Universidad de Sonora, dentro de su Catálogo de Servicios Profesionales para la Productividad se cuenta con un total de 112 servicios profesionales, de los cuales 12 se encuentran directamente relacionados con el cuidado del ambiente y desarrollo sustentable [16]. Por lo que, realizando el cálculo del indicador, como el número de servicios orientados al cuidado del medio ambiente y desarrollo sustentable del Catálogo de Servicios Profesionales de la UNISON entre el total de servicios ofrecidos multiplicado por 100, se obtuvo que el $10.71 \%$ de los Servicios Profesionales de la Dirección de Vinculación y Difusión de la UNISON se encuentra relacionado con el cuidado del medio ambiente.

\section{DISCUSIÓN}

Los resultados obtenidos en la evaluación de los indicadores para medir la contribución para la sustentabilidad en la UNISON, muestran el interés de la institución para aportar en el campo de la sustentabilidad. Destaca el hecho de que el $100 \%$ de las divisiones académicas que la conforman incorporan la perspectiva ambiental y de sustentabilidad al quehacer de la universidad como una política institucional, contribuyendo así con la mejora de la comunidad, la sociedad en su conjunto, el medio ambiente y el entorno en el cual se encuentra inmersa [17].

En lo que respecta a la perspectiva de género, es necesario que las instituciones de educación superior realicen la implantación de análisis institucionales serios con perspectiva de género y la puesta en práctica de una serie de estrategias para buscar una verdadera cultura 
de género que asegure una igualdad de oportunidades en el ámbito académico para mujeres y hombres [18]; considerando lo anterior, la UNISON se encuentra implementando el Programa Integral sobre Perspectiva de Género en la Universidad de Sonora [19], cuyos resultados han permitido que la institución cuente con un $32.26 \%$ de puestos funcionarios en la toma de decisiones representado por el sexo femenino; logrando así ir avanzando hacia una cultura de igualdad y respeto hacia la diversidad.

En cuanto al panorama educativo, se considera que la Universidad de Sonora es una institución comprometida con la enseñanza y difusión del desarrollo sustentable, ya que el $51.11 \%$ de su oferta educativa de licenciatura cuenta con materias relacionadas con el medio ambiente y el desarrollo sustentable; sin embargo, impacta el hecho de que solamente el $15.56 \%$ de los planes de estudio de nivel licenciatura se encuentren reconocidos por la institución bajo el enfoque de competencias, hecho que contrasta con lo establecido por el Decenio de las Naciones Unidas de la Educación para el Desarrollo Sostenible [1], donde se establece que la educación debe impartirse bajo este enfoque.

\section{CONCLUSIONES}

La Universidad de Sonora ha asumido su compromiso por cumplir con los lineamientos del Decenio de las Naciones Unidas de la Educación para el Desarrollo Sostenible, tal y como lo demuestran los indicadores que fueron evaluados dentro de este estudio; sin embargo, existen áreas de oportunidad donde la institución pudiera mejorar su desempeño, tales como el aumentar el número de proyectos de servicio social relacionados con el desarrollo sustentable, permitiendo así el aumentar su contribución de acuerdo con lo establecido con el Decenio mencionado.

\section{BIBLIOGRAFÍA}

[1] UNESCO (2007). The UN Decade of Education for Sustainable Development (DESD 2005-2014), The first two years. Disponible en: <http://unesdoc.unesco.org/ images/0015/001540/154093e.pdf> [Fecha de consulta 21 de Junio del 2013].

[2] Hernández, M.A., 1999. El cuidado del medio ambiental: análisis, reseñas, propuestas, crónicas, tesis, concepciones y paradigmas. [e-book] Edo de México: Universidad Autónoma del Estado de Mexico. Disponible en: Google Books http:// books.google.es/books?id=tKzlnQxHOPAC\&pg=PA34\&dq= problemas+ambientales \&hl=es\&sa $=X \& e i=r X b o U b T M G Y L c$ 9AT66YGoCg\&ved=0CEQQ6AEwAzgK\#v=onepage\& $q=$ pro blemas\%20ambientales\&f=false [Fecha de consulta 21 de Junio del 2013].

[3] Bory-Adams, A., 2006. UNESCO's role, vision and challenges for the UN Decade of Education for Sustainable Development (2005-2014). UNESCO International Science, Technology \& Environmental Education Newsletter, 31, 1-5.

[4] Hopkins, C., y McKeown, R. 2002, “Education for sustainable development: an international perspective" [online], En: Tilbury, D., Stevenson, R., Fien, J., Schreuder, D. Education and sustainability responding to the global challenge, IUCN Publications Services Unit. Disponible en: http://ibcperu. org/doc/isis/13028.pdf [Fecha de consulta 23 de Noviembre del 2013]

[5] SEMARNAT, s.f. Consejo Nacional de Educación Ambiental para la Sustentabilidad, [online] Disponible en: http://www. semarnat.gob.mx/educacionambiental/cneas/Paginas/ comoseformo.aspx [Fecha de consulta 3 de Julio del 2013]

[6] SEMARNAT, 2006. Estrategia de educación ambiental para la sustentabilidad en México (Versión ejecutiva, 20062014) [online] Disponible en: http://www.semarnat.gob. $\mathrm{mx} /$ educacionambiental/cneas/Documents/Estrategia\%20 de\%20Educación\%20Ambiental\%20para\%20la\%20 Sustentabilidad \%20-\%20SEMARNAT\% $202006 \% 20$ versión\%20ejecutiva.pdf [Fecha de consulta 3 de Julio del 2013]

[7] Filho, W.L., 2009. La educación para la sostenibilidad: iniciativas internacionales, Revista Educación. [pdf] Disponible en: http://www.revistaeducacion.mec.es/re2009/re2009_12. pdf [Fecha de consulta 16 de Diciembre del 2013]

[8] Gutiérrez, J., Benayas, J., Calvo, S., 2006. Educación para el desarrollo sostenible: Evaluación de retos y oportunidades del decenio 2005-20014, Revista Iberoamericana de Educación. Disponible en: http://www.rieoei.org/rie40a01. pdf [Fecha de consulta 18 de Diciembre del 2013]

[9] Garza, G.R, Medina, T.J.G., 2010. La Sustentabilidad en las Instituciones de Educación Superior: Una Visión Holística. Monterrey: LA\&GO Ediciones.

[10] UNISON, 2012b. Política de sustentabilidad. Disponible en http://www.sustentabilidad.uson.mx/ politicadesustentabilidad.html [Fecha de acceso 24 de Marzo del 2014]

[11] UNISON, 2014a. Otorgan el ISO 14001:2014 a Unison por acciones del Plan institucional de Desarrollo Sustentable. Disponible en http://www.uson.mx/noticias/default. php?id=16995 [Fecha de acceso 10 de Julio del 2014]

[12] [1] UNISON, 2011a. Directorio de funcionarios. Disponible en http://www.uson.mx/institucional/directorio/ [Fecha de acceso 24 de Mayo del 2014]

[13] UNISON, 2011b. Oferta educativa programas académicos. Disponible en http://www.uson.mx/oferta_educativa/ default.htm [Fecha de acceso 25 de Mayo del 2014]

[14] UNISON, 2013a. Dirección de innovación educativa. Disponible en http://www.innovacion.uson.mx/ [Fecha de acceso 16 de Marzo del 2014]

[15] UNISON, 2014b. Servicio social universitario. Disponible en http://www.serviciosocial.uson.mx/ [Fecha de acceso 13 de Marzo del 2014]

[16] UNISON, 2014c. Catalogo universitario de servicios profesionales. Disponible en $\quad$ http://www. vinculacionydifusion.uson.mx/serviciosprofesionales2014/ index.HTML\#/4/ [Fecha de acceso 16 de Mayo del 2014]

[17] Consorcio Mexicano de Programas Ambientales Universitarios para el Desarrollo Sustentable (COMPLEXUS), 2013. Indicadores para medir la contribución de las instituciones de educación superior a la sustentabilidad. [e-book] México: Universidad de Guanajuato. Acceso a través de: Academia Nacional de Educación Ambiental. Disponible en: http://anea.org.mx/ [Fecha de consulta 15 de Marzo del 2014]

[18] Verea, P., 2004. La política de género den la educación superior [pdf] Disponible en: http://148.202.18.157/sitios/ publicacionesite/pperiod/laventan/Ventana21/7-43.pdf [Fecha de consulta 5 de Septiembre del 2014]

[19] UNISON, 2012c. Impulsan Unison y CEDH Programa Integral sobre Perspectiva de Género, [online] Disponible en: http:// www.uson.mx/noticias/default.php?id=12914 [Fecha de consulta 8 de Septiembre del 2014]. 